AN EGG-TRANSFER STUDY OF EMBRYO SURVIVAL

AND MATERNAL PERFORMANCE IN FINN, GALWAY ANE FINGALWAY SHEEP

J. P. Hanrahan and J. F. Qutrike. - The Agricultural Institute, Belclare, Tuam, Co. Galway, Ireland.

The litter sizes of Finn, Galway and Fingalway ewes 2.5, I.4 and 2.0, respectively. Two or three fertilised eggs were transferred to recipient ewes of each breed. There was no difference among the breeds in pregnancy rate, egg-survival or litter size. Egg-survival and litter size were significantly lower when only two eggs were transferred. Maternal performance was measured by lamb birth weight and growth rate from birth to eight weeks of age. When these variables were expressed relative to ewe metabolic body weight there were no significant differences among the recipient breeds. The implications of the results for selection for litter size and for breed development and crossing using the Finn breed are discussed.

\title{
Fruchtbarkeit, Aufzuchtleistung und Wachstum von Schafen UNTERSCHIEDLICHEN FinNANTEILS UNTER BERÜicKSICHTIGUNG Der Haemoglobin- und Blutkaliumtypen
}

W. Hartmann und R. Wassmuth. - Institut für Tierzucht und Haustiergenetik der JustusLiebig-Universität Giessen, BRD.

Durch Einkreuzung von Finnschafen $(F i)$ in schwarzköpfige Fleischschafe $(s F)$ stcigt in einer Stichprobe von 300 Schafen die Genfrequenz für $\mathrm{HbA}$ von $8,3 \%(\mathrm{sF})$ auf $77,5 \%$ (Fi $\times$ $(\mathrm{Fi} \times \mathrm{sF})$ ), die für $\mathrm{K}^{h}$ von $20,4 \%$ auf $78,7 \%$.

Bei den untersuchten 2-7 jährigen Schafen ist die $F_{1}$ den $s F$ im Ablammergebnis um 79,6 Lämmer je ıoo Mutterschafe $(220,6 \%$ : I 4 I, 0I \%) überlegen $(p<0$, OOI), wobei ohne mutterlose Aufzucht die Differenz bei 90 Lebenstagen der Lämmer auf 32,2 verringert wird. Kreuzungstiere unter Verwendung von Milchschafen $(\mathrm{Fi} \times \mathrm{Mi}) \times \mathrm{sF}$ verbessern das Aufzuchtergebnis relativ stärker als das Ablammergebnis. Es zeigt sich eine Tendenz, daß HbAA-Schafe geringere Aufzuchtziffern und LK-Tiere eine bessere Gewichtsentwicklung haben.

Bei mit I 4-I 5 Monaten lammenden Schafen hatten Rückkreuzungen auf $\mathrm{Fi}(\mathrm{Fi} \times(\mathrm{Fi} \times \mathrm{sF}))$ die höchsten Ablammergebnisse ( $178,9 \%)$, die $\mathrm{sF}$ ( $133,3 \%)$ die geringsten $(p<0,00 \mathrm{I})$. Die Differenzen zwischen $F_{1}, F_{2}, R_{1} \mathbf{F} i$ und $R_{1 ; F}$ sind nicht signifikant.

\section{STHigerUNG DER FRUChTBARKEIT DURCh GEBRAUCHSKREUZUNGEN MIT FINNSCHAFEN}

\section{E. Kallweit, BRD.}

Die Rassen Deutsches Schwarzköpfiges Fleischschaf une Texelschaf wurden mit Finnischen Landschafen gekreuzt, um die Reproduktionsleistung ihrer weiblichen Nachkommen zu erhöhen. Der Vergleich von Ablamm- und Aufzuchtergebnissen sowie Wurfgewicht zwischen den Rasseund Kreuzungsgruppen et folgte unter gleichen Bedingungen bei Koppelschafhaltung.

Die Prüfung der verschiedenen Ein flußfaktoren ergat, daß Ablamm- und Aufzuchtergebnisse hauptsächlich von der Rassezugehörigkeit der Mutterschafe beeinflußt wurden, während das Wurfgewicht darüberhinaus auch von Versuchsjahr (1967-1972), Geburtsmonat und Rasse des Deckbockes abhängig war.

Nach der Korrektur für alle geprüften Einflußfaktoren errechnete sich für reine Finnschate ein Ablammergebnis von $238 \%$ (bei roz Ablammungen), für Schwarzköpfige Fleischschafe von I $56 \%(n=195)$ und für Texelschafe von $\mathrm{I}_{44} \%(n=\mathrm{I} 3 \mathrm{I})$.

Die Kreuzungsmutterschafe Finnschaf $\times$ Schwarzkopfschaf $(n=89$ bzw. 9) überstiegen das mittlere Ablammergebnis zwischen beiden Ausgangsrassen um I $5 \%$. Für die Kreuzungen zwischen Finnschaf und Texelschaf $(n=44$ bzw. 37) betrugen die entsprechenden Zahlen $12 \%$ bzw. $18 \%$.

Die Aufzuchtergebnisse stiegen mit zunehmendem Ablammergebnis an, obgleich auch die Aufzuchtverluste zunahmen.

Die Wurfgewichte waren beim Finnschaf aufgrund der geringen Geburtsgewichte der einzelnen Lämmer und bei den Kreuzungen Texelschaf $\times$ Schwarzkopfschaf aufgrund des niedrigen Ablammergebnisses geringer als in den übrigen Gruppen. 